\title{
Does having a doctor present on every medical ward on a bank holiday improve escalation and timeliness of review of a patient with a high national early warning score (NEWS)?
}

\author{
Authors: Olivia Dow and Tahir Akbar
}

\section{Aims}

This project aimed to evaluate the improvement of patient safety when having a doctor present on every medical ward on a bank holiday by evaluation of the use of the NEWS. Its implementation was reviewed in terms of timeliness and efficiency of care escalation and adherence to the guidelines.

\section{Methods}

We initially collected data on timeliness of escalation and review on all of our medical inpatients who had a NEWS score greater than 7 over the course of 1 month. We found that $50 \%$ of patients did not have appropriate escalation of their NEWS, as no initial call was made once the observations were done. We conducted a detailed causative analysis with individual groups involved in the escalation pathway, and felt that the presence of an identifiable doctor was more likely to prompt escalation of a NEWS score.

There were concerns that cover for 12 medical wards on a bank holiday consisted of only a foundation year one doctor and core medical trainee and therefore patients may not be appropriately escalated.

We changed our medical rota by having one doctor with at least 2 years' experience on each medical ward on a bank holiday. We collected data on escalation and timeliness of review in response to a NEWS score over 7 and reviewed individual cases.

\section{Results}

On the bank holiday, $98 \%$ NEWS scores were escalated and $89 \%$ were seen by a doctor. In comparison to data collected over a month, $40 \%$ of NEWS scores over 7 had been escalated and only $35 \%$ of patients were reviewed by a doctor. Feedback was positive, with the majority of doctors willing to continue this pattern of working for all foreseeable bank holidays.

Authors: Hampshire Hospitals NHS Foundation Trust, Hampshire, UK

\section{Conclusions}

The data support the theory that doctor presence on a ward meant that a high NEWS score was more likely to be escalated and reviewed in a timely manner, as it served as a reminder. We believe the presence of a doctor was a prompt to escalate a high NEWS score and, as it is well-known early recognition of deteriorating patients, improves patient outcome. Further work should focus on identifying factors that prevent high NEWS scores from being escalated.

\section{Conflict of interest statement}

No conflict in interest. 